\title{
Hinweise zur Benutzung
}

Die Bibliographie zu den Jüdischen Schriften aus hellenistisch-römischer Zeit (JSHRZ) stellt Editionen, moderne Übersetzungen, Monographien, Aufsätze, Lexikonartikel und Rezensionen zu allen in die JSHRZ aufgenommenen Schriften zusammen. Die Anordnung der Einzelbibliographien erfolgt in Anlehnung an die Reihenfolge des Erscheinens der Einzellieferungen. Den Bibliographien zu den einzelnen Schriften voran steht ein Abschnitt, in dem Titel aufgeführt werden, die einführenden Charakter haben: Eine "Bibliographie der Bibliographien" gibt zunächst einen Überblick über Hilfsmittel, die für die Erstellung der Einzelbibliographien ausgewertet wurden. Hiernach findet sich eine Liste mit Einleitungen zu den JSHRZ sowie eine Zusammenstellung wichtiger methodologischer und forschungsgeschichtlicher Beiträge, die zumeist wertvolle Spezialbibliographien enthalten. Der erste Teil wird mit einer Liste der wichtigsten wissenschaftlichen Übersetzungen der Apokryphen und Pseudepigraphen und einer kurzen Zusammenstellung einiger wichtiger Hilfsmittel (Konkordanzen und Indizes) abgerundet.

In einem zweiten Abschnitt, "Literatur zu übergreifenden Themen", werden solche Titel aufgeführt, die sich keiner der in die JSHRZ aufgenommenen Schriften allein zuordnen lassen, in einer Bibliographie zu den JSHRZ jedoch nicht fehlen sollten. Einige der in diesem Abschnitt aufgeführten Titel sind auch in den Einzelbibliographien berücksichtigt worden. Da die in diesem Abschnitt der Bibliographie aufgeführten Titel zumeist Einleitungs- oder Überblickscharakter besitzen, werden sie auch hier aufgelistet.

Die Gliederung der Einzelbibliographien erfolgt in Anlehnung an die Literaturverzeichnisse der bisher erschienenen Lieferungen der JSHRZ: Den wichtigsten "Textausgaben" (a), die nach Herausgeber oder Bearbeiter alphabetisch - innerhalb des Alphabets chronologisch - geordnet sind und zu denen auch Textkonkordanzen und Synopsen und grundlegende Textausgaben mit kommentierten Übersetzungen gezählt werden, folgt unter (b) eine Zusammenstellung der wissenschaftlichen Übersetzungen und Kommentare bzw. kommentierten Übersetzungen. Nur in der Bibliographie zu Joseph und Aseneth wurden nach C. Burchard (JSHRZ II / 4) weitere Rubriken eingeführt. Ansonsten werden "Tochter-" und "Enkel"-Übersetzungen sowie "sekundäre" Textzeugen, die zuweilen die einzigen Zeugen für die Rekonstruktion einer Schrift darstellen, je nach Stand der Forschung, unter (a) aufgeführt. Um den 
Überblick über die wichtigsten Textausgaben zu erleichtern, werden Vorveröffentlichungen einzelner Textfragmente (insbesondere solcher aus der Wüste Juda) und sekundäre bzw. tertiäre Textzeugen nicht immer nur unter (a) oder (b) aufgeführt, sondern zuweilen auch, wie alle weiteren Einträge, in der Rubrik (c): "Aufsätze - Artikel - Monographien".

Um die mehrfache Aufführung von Titeln weitestgehend zu vermeiden, sind Aufsätze, Artikel und Monographien, die mehrere Schriften behandeln, nur dann wiederholt aufgeführt worden, wenn sie entweder im Titel auf mehrere der betreffenden Schriften hinweisen oder so grundlegenden Charakter haben, daß ihre mehrfache Auflistung unumgänglich erscheint. Einige Einträge, die man z. B. in den Bibliographien zum 2. oder 4. Makkabäerbuch vergeblich sucht, wird man daher nur in der Liste zum 1. Makkabäerbuch finden. Vergleichbar ist auch für die Bibliographien zu den Testamenten oder zum syr. Baruch und dem 4. Esra-Buch zu verfahren.

Sind hinter einem mehrfach aufgeführten Titel Seitenangaben vermerkt, beziehen sich diese Angaben stets auf einen besonderen ("bes.") Abschnitt oder ein spezielles Kapitel, das sich vorwiegend oder ausschließlich mit der jeweiligen Schrift befaßt. Einträge, hinter denen sich keine speziellen Seitenangaben finden, enthalten kein besonderes, die jeweilige Schrift behandelndes Kapitel, erwähnen diese Schrift allerdings so häufig, daß auf ihre Berücksichtigung in der Bibliographie nicht verzichtet werden konnte.

Titel aus der Zeit vor 1800 werden nur in Ausnahmefällen aufgeführt. Da jedoch die Berücksichtigung von Veröffentlichungen aus der Zeit vor diesem Datum für einige Schriften unumgänglich ist, ist die zeitliche Begrenzung nicht immer strikt eingehalten. Für die ältere Literatur sei generell auf den Abschnitt "Bibliographie der Bibliographien", insbesondere auf die umfassenden Bibliographien von J. Fürst (\# 29), Y. Vinograd (\# 79-80) und R. W. Thompson (\# 77) verwiesen.

Rezensionen sind nur teilweise erfaßt worden. Mit der Aufführung von Rezensionen zu besonders wichtigen Werken soll das wissenschaftliche Echo auf grundlegende Publikationen dokumentiert werden (vgl. z. B. \# 361). Eine auch nur annähernd vollständige Aufnahme der Rezensionen zu allen in Frage kommenden Einträgen erschien, insbesondere bei Sammelwerken und Festschriften, als wenig sinnvoll und in der zur Verfügung stehenden Zeit kaum durchführbar. Rezensionen sind stets in runden Klammern bei dem ersten Eintrag eines Titels vermerkt. Wird ein Titel in einer Bibliographie zu einer anderen Schrift öfters aufgeführt, sind die Rezensionen nicht noch einmal bei dem entsprechenden Titel verzeichnet.

Die Titelaufnahme weicht von dem bislang in den Lieferungen der JSHRZ gepflegten System ab: $\mathrm{Zu}$ beachten ist vor allem, daß sich alle Abkürzungen nach S. M. Schwertner, IATG ${ }^{2}$. Internationales Abkürzungsverzeichnis für Theologie und Grenzgebiete, 2. überarb. und erw. Aufl., Berlin [u. a.] 1992, richten. Die Abkürzungsverzeichnisse, die der ersten Lieferung eines Bandes der JSHRZ beigegeben sind, wurden nicht mehr berücksichtigt. Einige in 
Schwertners Abkürzungsverzeichnis noch nicht aufgenommene, mittlerweile gebräuchliche Abkürzungen wurden in das beigefügte, alle verwendeten Abkürzungen berücksichtigende Verzeichnis aufgenommen. Neuere Zeitschriftenoder Reihenbezeichnungen, für die sich noch keine Abkürzungen durchgesetzt haben, sind mit vollständigem Titel angegeben.

Zur Titelansetzung: Selbständige Schriften werden immer in folgender Form angegeben: Verfasser: Titel. (Untertitel), Reihe, Ort, Jahr. Ist ein Werk in einer Reihe erschienen, wird die Reihe nach dem Titel und vor dem Ort angegeben. Grundsätzlich wird die neueste Auflage genannt; nur bei einigen grundlegenden älteren Werken ist vor der neuesten auch die erste Auflage vermerkt. Der Hinweis auf einen Nachdruck steht in eckigen Klammern hinter der Jahresangabe. Nur bei einigen grundlegenden Titeln wurden auch die Übersetzungen ins Englische oder andere moderne Wissenschaftssprachen berücksichtigt.

Zeitschriftenartikel werden wie folgt angesetzt: Verfasser: Titel, Zeitschrift, Jahrgang (Jahr), Seitenangabe. Teilbeiträge in Sammelbänden sind folgendermaßen verzeichnet: Verfasser: Titel. (Untertitel), in: Fundort, Herausgeber, Reihe, Ort, Jahr, Seitenangabe. Bei Lexikonartikeln wird das Erscheinungsjahr des Bandes angegeben; auf die Angabe "Sp." (für Spalten) oder "S." (für Seiten) wurde verzichtet. Beiträge, die in Sammelbänden und Gesammelten Studien desselben Autors wiederveröffentlicht wurden, werden - ohne die Kürzel "ders." bzw. "idem" - aufgeführt. Nachdrucke von Aufsätzen und Rezensionen sind zuweilen in eckigen Klammern hinter dem Erstfundort vermerkt; ein vorangestelltes " $="$ deutet in all diesen Fällen an, daß der betreffende Eintrag von demselben Verfasser stammt oder in einem Sammelband, der unter demselben Verfassernamen veröffentlicht wurde, zu finden ist. Der Herausgeber eines Sammelbandes wird stets hinter dem Titel der Schrift angegeben.

Hat eine Schrift mehrere Herausgeber, wird nur der erste Herausgeber mitgeteilt, alle weiteren Herausgeber werden durch "u. a." ersetzt. Bei Sammelbänden und Schriften, zu denen sich kein Herausgebervermerk findet, wird auf dem Originaltitelblatt kein Herausgeber genannt. Dies ist insbesondere für einige französischsprachige Einträge zu beachten, in denen der (oder die) Herausgeber oft nur durch die Lektüre des Vor- oder Nachwortes zu ermitteln ist. In einigen dieser Fälle wurde der Herausgebervermerk in eckigen Klammern aufgeführt.

Festschriften ("FS") werden (i. d. R.) mit dem abgekürzten Vornamen und Familiennamen des Geehrten aufgeführt. Der Name des ersten Herausgebers findet sich wie bei Aufsatzsammlungen stets nach dem Titel der Festschrift. Handelt es sich um mehrbändige Festschriften (oder Aufsatzsammlungen), wird nur der erste Herausgeber des jeweiligen Bandes genannt.

Dissertationen werden nur dann gesondert aufgeführt, wenn das Werk nicht bereits an einem anderen Ort veröffentlicht worden ist. Übersetzungen oder Überarbeitungen einiger Dissertationen werden i. d. R. gesondert aufgeführt. 
Zwischen "Diss. theol." und "Ph. D." wurde dabei nicht unterschieden. Bei Dissertationen, die an einer Hochschule in den USA angefertigt wurden, wird die Universität als Erscheinungsort aufgeführt. Auf die Angabe des Bundesstaates oder der Stadt wird in diesen Fällen, wie auch in allen Fällen, in denen der Bundesstaat nicht auf dem Titelblatt hinter dem Erscheinungsort verzeichnet ist, verzichtet.

Bei der Interpunktion und Schreibung einzelner Worte wurden aus Gründen der Vereinheitlichung kleine Eingriffe vorgenommen. So sind bei Stellenbzw. Versangaben die Doppelpunkte oder Semikola zuweilen durch Kommata ersetzt worden. Auch auf die Wiedergabe von kursiven Lettern in der Originaltitelansetzung wurde verzichtet.

Hebräische, griechische und kyrillische Titel sind immer in Originalsprache aufgeführt. Die Ansetzung des Verfassernamens richtet sich in diesen Fällen entweder nach dem Paralleltitel oder nach der in weiteren Publikationen desselben Verfassers üblichen Namensform. Die Umschrift des Hebräischen (etwa bei Zeitschriftennamen, die keinen Paralleltitel besitzen) folgt dem Verfahren, das in FJB 2, 1974, 65-68, entwickelt und begründet wurde. Bei kyrillischen Titeln wird dem Originaltitel die Umschrift des Titels in eckigen Klammern nachgestellt. Die Umschrift des Russischen richtet sich nach der "Transkriptionstabelle der slawischen kyrillischen Buchstaben" in dem "Regelwerk der Alphabetischen Katalogisierung wissenschaftlicher Bibliotheken" (S. 427). Georgische Titel werden nach F. Zorell (\# 420) transkribiert.

Bei aller angestrebten Konsequenz bei der Vereinheitlichung der Titelansetzung, Schreibweise und Transkription war es gelegentlich nötig, Ausnahmen zu machen.

Das beigefügte Namensregister führt in alphabetischer Reihenfolge alle in der Bibliographie aufgenommenen Autoren, Herausgeber von Texteditionen und Übersetzer einzelner Schriften auf. Nicht berücksichtigt sind die Herausgebernamen von Aufsatzsammlungen, Festschriften oder die Namen der Verfasser von Rezensionen. 